\title{
Designing and Developing e-Assessment Delivery System Under IMS QTI ver.2.2 Specification
}

\author{
https://doi.org/10.3991/ijet.v16i01.16257 \\ Mohammed Boussakuk $\left.{ }^{(}\right)$, Ahmed Bouchboua, Mohammed El Ghazi, \\ Moulhime El Bekkali \\ Sidi Mohamed Ben Abdellah University, Fez, Morocco \\ mohammed.boussakuk@usmba.ac.ma \\ Mohammed Fattah \\ Moulay Ismail University, Meknes, Morocco
}

\begin{abstract}
Using technology to create online examination is important, more than that, exploring the recent research in the area of standardization of the learning and the assessment content is of paramount. However, there is still a lack of reusable, interoperable and exchangeable learning resources that afford much motivation to instructors. Assessing acquired knowledge is the primary task of any Learning Management Systems (LMS). Consequently, sharing evaluation contents is a major challenge for software engineering. In this paper, we present the "CleverTesting" assessment authoring tool, focusing on its architecture and its conformance to the Question and Test Interoperability (QTI) specification proposed by IMS Global Learning Consortium. We adopt the QTI specification to create reusable, interoperable and sharable assessment content. Our system has three important components: The first component is "CleverTesting" a quiz authoring tool that allows building sharable question items. The second represents a question bank. The latest component is a player for interpreting and parsing QTI XML documents.
\end{abstract}

Keywords-IMS-QTI, Question and Test Interoperability, e-Learning, eAssessment, Individualized Learning

\section{$1 \quad$ Introduction}

Nowadays, the use of Information and Communication Technologies (ICT) in education have fundamentally changed the way people learn and teach. So, electroniclearning (E-learning) or web-based instruction refers broadly to the use of ICT to deliver learning content electronically and to promote teaching strategies. Actually, eLearning has become a crucial segment of instruction environments [1]. As a significant research field, it has a great deal of interest and has grown considerably.

One of the more powerful phases of the e-learning process is online assessment (eAssessment). Assessment is the process of measuring the skills, abilities, the learning progress or educational needs of the learners' acquired knowledge during their learn- 
ing activity. The term Computer-Assisted Assessment (CAA) refers to the use of digital technologies to distribute evaluations, correct and storage responses [2].

In recent years, several online testing systems have been developed, either free or commercial. As a result, many of the assessment content have been produced and stored in repositories. These systems generally use proprietary coding and storage formats to build the questions and tests, which is an expensive, time and energy consuming task. This proliferation and diversity of tools lead to the problem of the durability, interoperability and reuse of these educational objects [3]. In this context, several organizations and consortiums are working on the development of education specifications and standards to unify and ensure the interoperability of distributed education applications and services.

For these reasons, the development of tools for creating items and tests in accordance with educational norms and standards are issues of concern. The ultimate goal of this document is to propose the design and development of an electronic assessment tool, called "CleverTesting", that allows instructors to build tests/quizzes and items and items in accordance with the latest version of the IMS Question and Test Interoperability (QTI) specification and accessible through different compliant systems.

In our team, we have previously designed and implemented a Dynamic Adaptive Hypermedia System (DAHS) called "CleverUniversity" [4]. This is an individualized and adaptive e-learning system where each student receives a personalized instruction content according to his or her knowledge level and learning style [5]. Our new "CleverTesting" module will be integrated into our "CleverUniversity" system, while considering its integration into other community projects like Moodle. This phase will be followed by experiments to improve our environment and evaluate its effectiveness.

The rest of this paper is structured as follows: The second section tackles the importance of online assessment and its categories. Moreover, it demonstrates the importance of e-learning specifications along with its standards. Furthermore, at the end of this section, we will have a short glance at the IMS-QTI specification and the reasons for its use will be given too. The third section defines some related works. The fourth section presents the architecture and design of the "CleverTesting" eassessment system and covers its different components. Finally, we conclude and provide some outlines for future work and research.

\section{$2 \quad$ Literature Review}

\subsection{The importance and varieties of e-assessment}

The activity of evaluation plays a crucial role in the learning process, it is an integral part of any effective educational system or program. The value of assessment is twofold, on the one hand, it allows learners to recognize their level of understanding during the learning process, to validate their knowledge and to detect their strengths and/or weaknesses in a particular subject [6]. On the other hand, it allows the teacher 
to monitor the progress of his or her learners, identify any difficulties and needs, in order to intervene effectively to provide help and remediation.

Some fundamental objectives of evaluation are:

- Guiding the teacher, the learner and the tutor throughout the learning process and help them to make the right decisions to improve the quality of learning [7].

- Checking the results obtained in relation to the initial objectives of the learning process. Following this diagnosis, the teacher can determine the current level of the learner and provide useful and effective feedback. And if necessary, he might create a remediation plan and some remedial work for learners with difficulties [6].

- Producing state-recognized certificates, diplomats and degrees.

Today, technology plays an important role in the evaluation process. According to some studies conducted on electronic assessment, the use of technology in the field of assessment has developed rapidly and has been adopted by educational institutions [8]. Joint Information Systems Committee (JISC) [2] defines e-Assessment as "the end-to-end electronic assessment processes where ICT is used for the presentation of assessment activity, and the recording of responses. This includes the end-to-end assessment process from the perspective of learners, tutors, learning establishments, awarding bodies and regulators, and the general public". E-Assessment is usually offered on learning environments or LMSs (Learning Management Systems) to produce tests and quizzes. These tools generally offer several types of questions, with or without multimedia files, which can be presented in a random order with a fixed number of attempts and a predetermined response time [9].

According to [10], three types of assessments can be found in an educational curriculum: Formative, summative and diagnostic (also known as pre-assessments).

- Formative Assessment: Assesses the quality of learning during and throughout the learning process. It provides useful feedback to both learner and teacher on a given topic or concept. This feedback makes it possible to detect the strong and weak points of each student, allowing the teacher to intervene effectively to remedy any anomaly during the learning phase [11].

- Summative Assessment: Takes place at the end of a learning program, the main objective of which is to judge the candidate's success or failure in relation to the initial objectives of the learning curriculum [12].

- Diagnostic Assessment: Generally undertaken at the beginning of a learning program or activity, which is used to identify a learner's skills and gaps in order to provide adequate tutoring and personalized feedback.

Generally, formative and diagnostic assessment help to improve the quality of teaching as well as the level of the learner [7]. Although technology has greatly facilitated the production of tests and items, the teacher should administer assessments only when he deems it necessary to improve the learning quality.

Another important issue is related to the difficulty of reusing valuable evaluation resources due to the lack of a common creation format among various assessment 
systems. The following section provides background information on the importance of educational standards in ensuring interoperability of electronic assessment content.

\subsection{An overview of IMS question and test interoperability}

Generally, producers of e-testing in the market use a proprietary coding format to write items and assessment, which require specific players for their deployment. The main goal of this project is to ensure interoperability and the sharing of these exercises and questions through different tools. Following a study of a variety of standards and interoperable question creation formats, the IMS-QTI specification is the most promising and successful standard in e-learning domain [13].

The IMS Question and Test Interoperability, or QTI, is one of the IMS Global Learning Consortium (IMS GLC) standards for the development of learning technology software. QTI identifies and defines the metalanguage and interoperability structures that saves time and money for the various actors in the implementation, distribution and outcomes of digital evaluations. In addition to that, QTI permits the exchanging and sharing items and/or complete tests and reporting their associated results between test authoring software, item repositories, test delivery tools and other QTI compliant systems, and scoring administrative systems [14] [15]. Moreover, the QTI specification is based on the Extensible Markup Language (XML) to describe question items. XML is a flexible markup language and a key technology for ensuring interoperability and extensibility of data. For these reasons, IMS-GLC adopts XML in all its standards. QTI specification can be used for simple quizzes as well as highstakes summative evaluations.

For interoperability, the QTI specification describes data model for the representation of question (assessmentItem) and test (assessmentTest) data and their corresponding results reports [16]. Therefore, this specification allows the interoperability of these different structures within assessment systems and different suppliers. This will be useful and helpful for many social actors such as content creators, vendors, schools, states, private institutions, as well as the software publishers whose tools they use and so many others.

To sum up, the IMS QTI is the only well-known and widely used format for exchanging assessment data independently from the authoring tool used to create them. In our research work, we use the latest update of QTI version 2.2, released on November 27, 2017, which defines more than 20 types of interactions (simple or complex items) [17] [18]. Due to their complexity, many of these types are rarely used. However, this complexity and richness of the specification lead us to develop authoring tools that are more intelligent and advanced to assist the teacher in his/her task in creating digital assessment content.

Figure1 shows a choice question with one point of interaction. The corresponding QTI-XML is shown in figure 2, this XML document includes the corresponding response processing as well as the display directives. Here the question used to obtain a single response from the candidate. 


\section{What is the capital of morocco ?}

$\mathrm{Fez}$

Rabat

Casablanca

None of the above

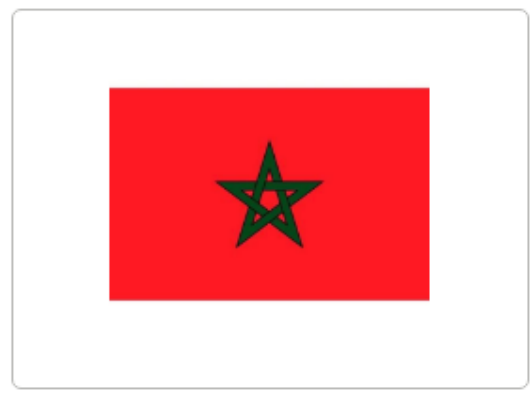

Fig. 1. A simple choice question.

The text of the question part (stem) of the item is shown in line 21 . The answer options are found on lines 22,23,24 and 25 . We can notice that the code required to write this simple item is very complicated, as illustrated by the example above. It does not yet include rules for handling feedback and custom responses. This would have made the document more confusing and enigmatic.

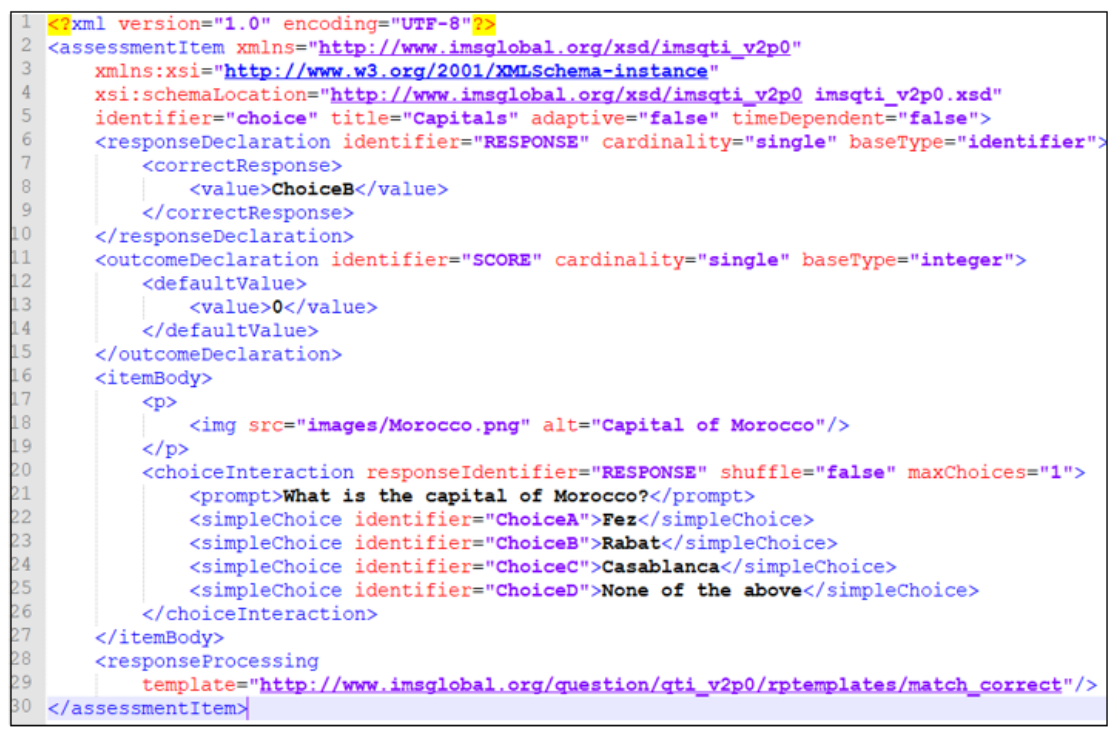

Fig. 2. A single choice question coded in IMS-QTI version 2

\subsection{Related works}

As has already been mentioned, it is common to find a variety of applications available for the development of digital assessment. However, most of them do not fully support the IMS-QTI specification and do not allow content evaluation interop- 
erability, according to a study conducted by [19]. Another study by [20] found that the majority of the applications analyzed failed to export/import IMS-QTI files. In addition to this, [21] it shows that the most recent e-learning systems use obsolete versions of the QTI specification to represent different assessment objects.

R2Q2 from the University of Southampton was the first response and rendering engine for QTIv2.0 questions. R2Q2 is a project founded by the Joint Information Systems Committee (JISC), but has been discontinued and is no longer maintained [22].

ATutor is an Open-Source Web-based LMS used to develop and deliver online courses and tests. Its adoption of QTI is limited to the import/export functionalities of QTI-XML files old version 1.2. According to a comparative study of the most used e-learning systems carried out by [23] in 2019, concludes that this system is not userfriendly from the users' point of view, in addition to which the creation of tests and their completion is a big problem. These same authors also show that the Moodle LMS lacks clarity when creating questions. These are created using Moodle's proprietary format called GIFT. Except that it allows you to import and export certain types of questions according to the QTI specification [23].

In 2019, Vomvyras and al. [24] proposed a web-based online examination system that enables authors to automatically generate tests using pre-written questions stored in a database. The system does not support the QTI specification and therefore does not improve the interoperability and reuse of evaluation objects.

In this paper, "CleverTesting", a web-based online assessment tool incorporating many of the missing features is presented. CleverTesting is a free and open-source application to assist instructors in building reusable assessment items and tests in accordance with the latest version of QTI Specification 2.2.2 (27 November 2017).

\section{CleverTesting: A Novel e-Assessment System}

\subsection{The Features of CleverTesting}

CleverTesting solves many of the aforementioned issues, namely:

- It is based on the latest version of the QTI 2.2.2 specification, while providing for rapid migration to the new version 3.0 which should be released in the coming months.

- It helps evaluators to create tests that are compliant with QTI specifications without having any special technical knowledge.

- It supports a variety of question types.

- It provides permission roles for teachers to fully manage learner's activities.

- It uses real-time events and timers when tests are scheduled to begin.

- It disconnects students when the timer expires without losing their answers.

- It provides teachers with ready-to-use exercise templates. 


\subsection{An overview of the architecture}

This section deals with CleverTesting architecture based on a client/server model. CleverTesting uses powerful technologies and frameworks that can support multiusers and parallel exams without impacting the user's experience. It was designed and encoded using Laravel PHP framework v7.10, Vue.js v2.6, Sass, Bootstrap v4 and MySql database. Laravel is based on MVC architecture which allows a low coupling between models, views or controllers.

CleverTesting can be examined under seven main parts. These are architecture, IMS-QTI Editor, Item Bank, Assessment Engine, Domain model and Learner model.

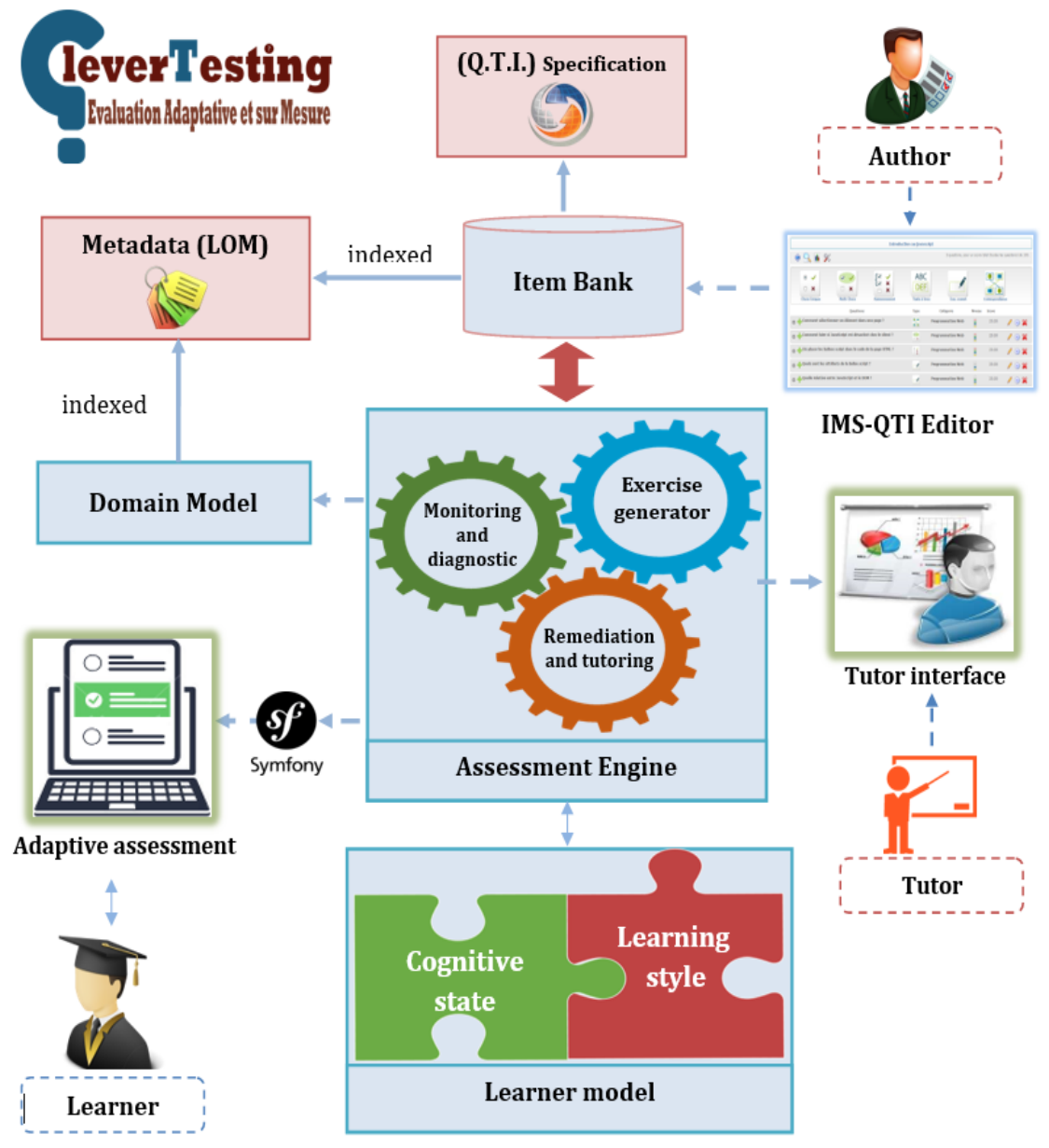

Fig. 3. «CleverTesting» Architecture overview.

Figure 3 above illustrates the basic architecture of our CleverTesting environment. It was developed as an intelligent e-assessment platform to assist teachers in building 
interoperable and reusable assessments and items in accordance with the latest version of IMS QTI Specification. The system provides a user-friendly editor to avoid the technical complexity behind the QTI specification. It is essentially based on five modules that work closely together: Domain model, student's model, assessment engine, item bank and QTI editor.

Regarding the domain model, it maintains the concepts used in learning that are created at the lowest level of granularity to ensure reusability regardless of the format used to build them. As for the student model, we are very interested in the reliability of the cognitive level of the student. It gives information about the learner's knowledge, objectives, personal traits and episodic memory. Therefore, this module is crucial for providing adapted teaching. The assessment engine carries out adaptive tests in accordance with the cognitive state of each student based on the information from the two previous modules. It comprises three components: i) a semi-automatic exercise generator. ii) a monitoring and diagnostic module enables observation, collection, storage and analysis of data resulting from the learner's interaction with our environment. iii) a tutoring module that provides personalized support and immediate feedback to the students. On the other hand, it allows educators to analyze and infer the learner's skills and to regulate his/her progress if necessary.

\section{$4 \quad$ System Analysis and Design}

\subsection{Class diagram of the main actors of the system}

Conceptually, electronic evaluation involves a number of systems and various actors. In fact, systems and individuals often play multiple roles; for example, in universities, instructors also play the role of test writers, monitors, and scorers.

Figure 4 shows the main actors in our system. The set of roles identified in this diagram has been reduced to a small set of abstract actors for simplicity. Each of these actors has different access rights according to their tasks. These roles are:

- Author: That can create, search or modify questions by using an authoring tool.

- ItemBankManager: The person responsible for managing a set of questions stored in itemBank.

- TestConstructor: His role is to create tests from the questions stored in itemBank.

- Proctor: Also known as invigilator, he is the person responsible for monitoring the progress of an assessment.

- Scorer: He can be a person or an automatic scoring system that evaluates candidates' answers.

- Tutor: Someone involved in the management, control or support of a learner's learning process.

- Candidate: The person being evaluated by an assessment element.

- Administrator: The person who manages the user and the group accounts. Also, it is responsible for managing the security of all information in the application.

- WorkflowInstructor: Generally, an item that can have only one author. However, in some cases, an item may be through a control and a validation process involving many people. 


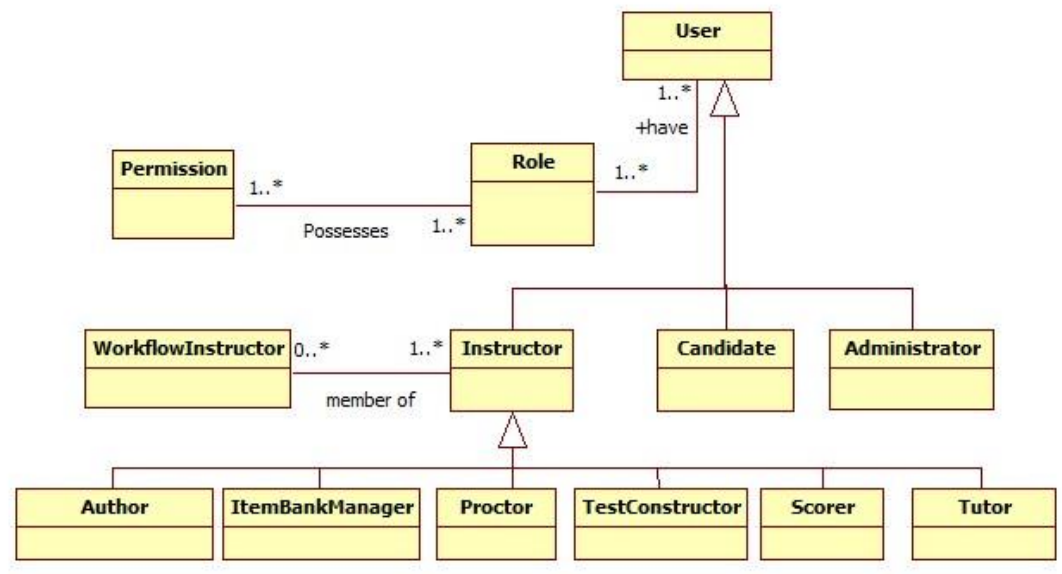

Fig. 4. Class diagram of the main actors of the system.

\subsection{Evaluation package}

The figure 5 below details and describes the different components of the evaluation package. This package allows instructors to create, modify and administer evaluations, just as it allows candidates to take these tests.

The core of this package is the AssessmentItem class which contains the main characteristics of a question. In other words, an item includes all the data needed to create, render, grade, and provide feedback from questions. These items may be grouped into sections (AssessmentSection); that themselves may contain other logically related sections; and finally, tests (AssessmentTest) are assembled from these sections.

For the majority of LMSs, there are common types of questions, among the most commonly used ones are: true/false question, Multiple Choice, matching or short answer questions. Our model can model most of these questions. The classes responsible for processing the items are ResponseProcessing, OutcomeProcessing and TemplateProcessing. ResponseProcessing assigns results as a function of the respondent's answers and according to a sequence of rules executed by the response processing system.

It is essential to give useful feedback to the candidate in order to improve the learning process. This feedback is generated following the candidate's answers, and is stored in our system for later use either by the instructor or by the learner. 


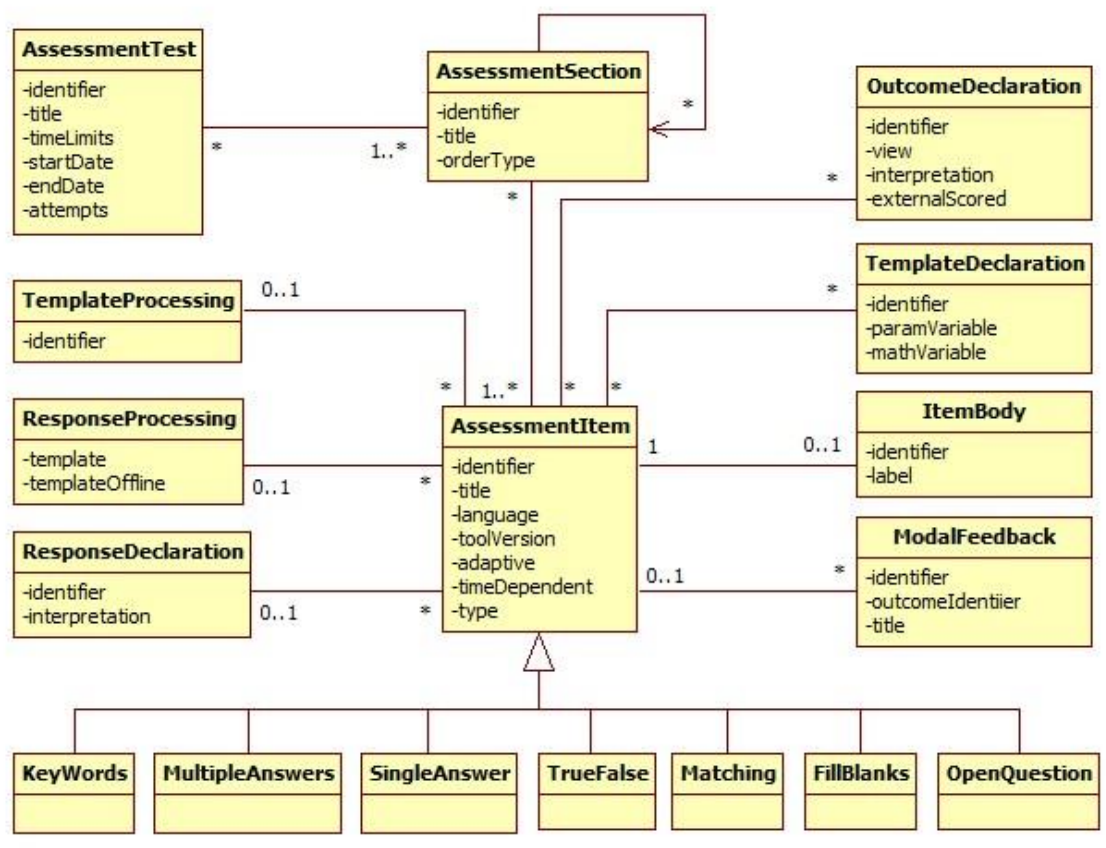

Fig. 5. Testing module in CleverTesting.

\section{The IMS-QTI Editor}

The IMS-QTI editor is a client / server architecture-based web application which can be accessed by the user via a web browser. Our authoring tool provides an intuitive and user-friendly interface for the author to simplify the creation of questions and tests, thus avoiding the technical complications behind QTI representation.

In other words, the instructor can generate new questions based on the IMS-QTI specification using this editor, or restore them using a multi-criteria search from the item Cache. In e-learning networks these things can be downloaded, reused, and exchanged. Furthermore, this tool ensures the availability of import/export evaluation content from/to XML files that conforms to IMS-QTI standard.

Figure 6 shows an interface for creating a test. The instructor can organize the items by category, specify the order in which they will appear, and specify the date and duration of the test. Candidates must register in order to take assessments and to receive notifications about scheduled tests or scores. Tests are stored in the database and can be accessed by instructors and learners at any time. 


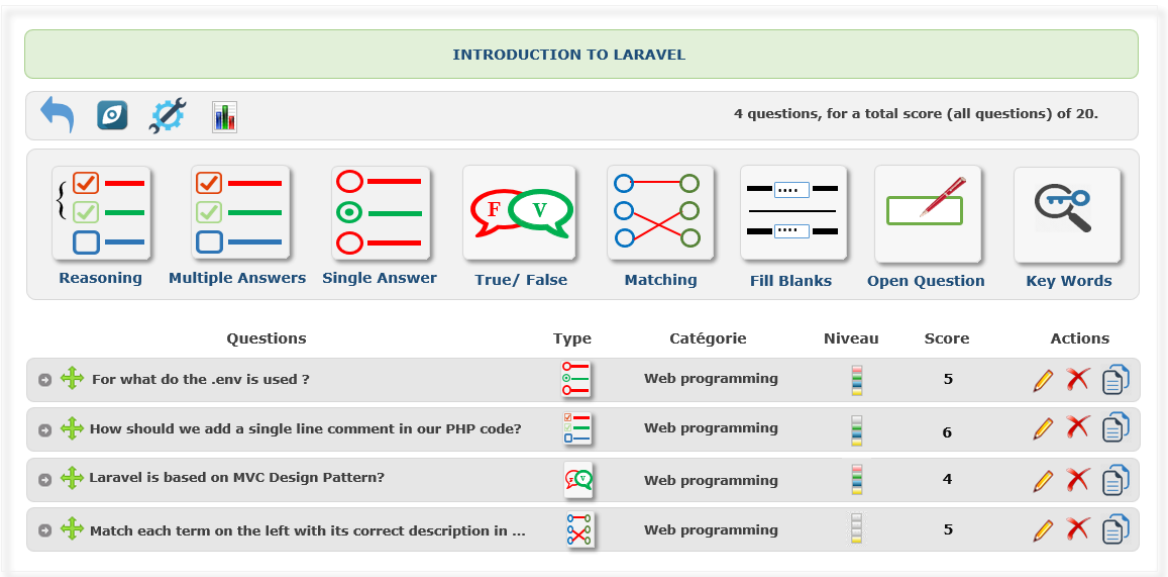

Fig. 6. The instructor creates a test via a user-friendly editor.

As shown in the figure 7 , the process of creating question with a single choice answer is too simple and flexible. First, the instructor writes the question's title, then adds the alternatives correct answers and determines the correct one. He can also provide valuable feedback for either the correct answer or the wrong answer.

Figure 8 shows the candidate's views of previously produced single-choice questions (figure 7). In this case, the tool displays the remaining time if the test is timelimited. Otherwise, the examinee can stop the evaluation and continue it later. Once the evaluation is completed, the examinee can review his answers, consult her/his score. Moreover, he/she may analyse the final feedback to improve her/his learning.

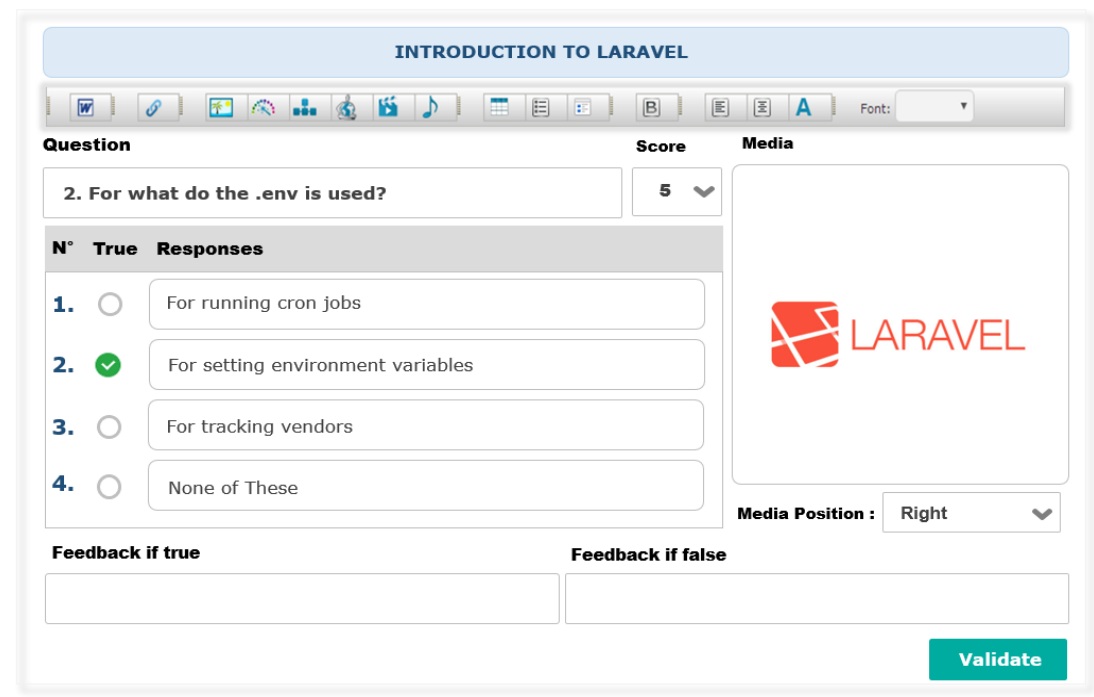

Fig. 7. Building a single choice questions in CleverTesting. 


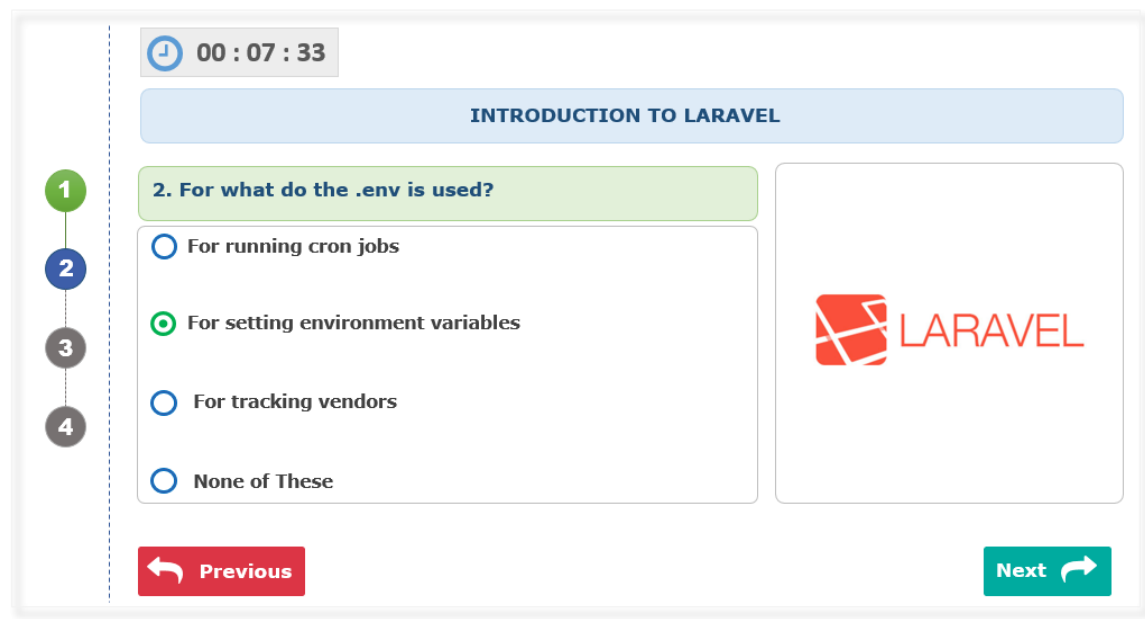

Fig. 8. Candidate's view of a single choice questions.

\section{The Communication Module}

Our e-Assessment environment offers asynchronous and synchronous communication tools. These online communication tools engage students in online interactions with a human teacher or peers. In addition, they provide support for group discussions, student collaboration and resource sharing. These include the messaging module, the real-time chat tool, the use of discussion forums and video conferencing (figure 9).

The messaging module was designed in order to provide messaging between members of CleverTesting. All the users registered in the platform can send emails to each other. The module was developed as a real Web-based e-mail service.

As mentioned above, our system is composed of a remediation and tutoring module that allows us to generate a personalized feedback report for each learner. The objective of this phase is to identify the procedural knowledge, correct or erroneous, that the learner has used when solving the exercises and to identify any difficulties during his learning progression. As a result, the teacher can easily answer the learner's questions, guide and coach him/her on a regular basis. In this context, the use of these different forms of communication promotes learner participation, increases their motivation and helps them achieve their learning objectives more effectively. 


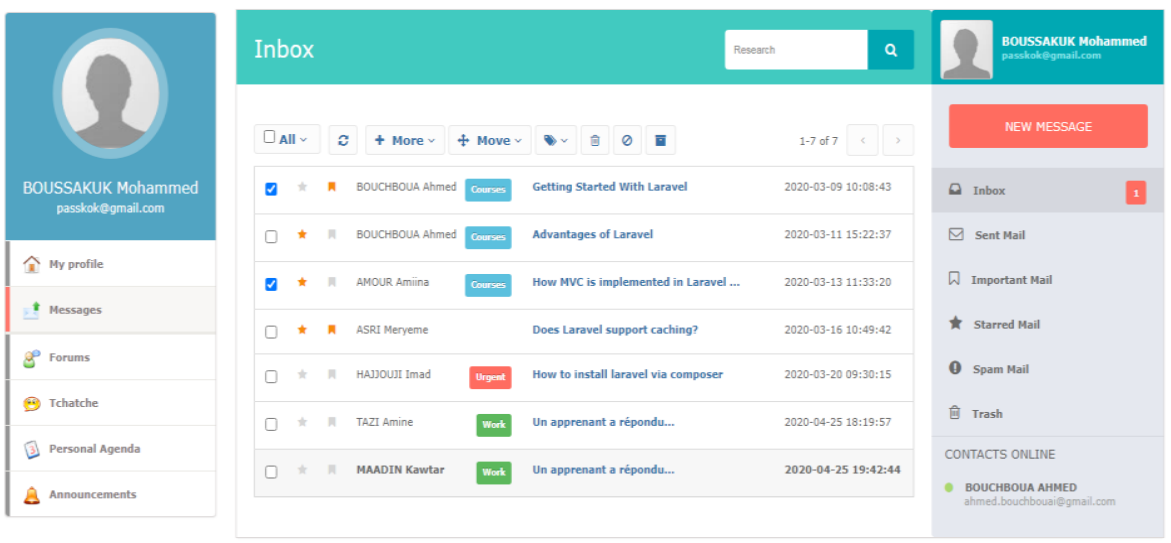

Fig. 9. Sample screen shot from the CleverTesting messaging module.

\section{$7 \quad$ Conclusions and Future Work}

One of the most tiring tasks in the learning process is to assess the learners. Our research looks at the computerized adaptive testing system (CAT) design and development process. In this paper, we addressed the first phase of our research on designing and developing an authoring system to help teachers build reusable evaluation objects according to the QTIv2.2 specification. Using this specification, the assessment contents will be easy to reuse, exchange and share among compliant systems. Since no special skills in the IMS-QTI specification is required, the instructor finds no difficulty in using our tool.

Our system is currently used only for testing. As future research, we plan to add new types of questions that the tool will create. We are also interested in applying Item Response Theory (IRT) to produce adaptive tests according to the ability of each examinee. In the long run, we are planning to integrate this tool into our experimental DAHS called CleverUniversity [4], and other e-learning systems, in order to test its effectiveness in real context.

\section{$8 \quad$ References}

[1] M. Halim, N. Adadi, D. Chenouni, and M. Berrada, "Web Services Composition in ELearning platform,” Int. J. Emerg. Trends Eng. Res., vol. 8, no. 2, pp. 525-532, 2020. https ://doi.org/10.30534/ijeter/2020/41822020

[2] Joint Information Systems Committee (JISC), "Effective Practice with e-Assessment," 2007.

[3] V. Tomberg, P. Savitski, P. Djundik, and V. Berzinsh, "Design and Development of IMS QTI Compliant Lightweight Assessment Delivery System," in Joosten-ten Brinke D., Laanpere M. (eds) Technology Enhanced Assessment, vol. 653, Springer, Cham, 2017, pp. 159-170. https://doi.org/10.1007/978-3-319-57744-9

[4] M. Boussakuk, A. Bouchboua, M. E. L. Ghazi, M. Fattah, and M. E. L. Bekkali, “A Fully individualized Adaptive and Intelligent Educational Hypermedia System: Details of 
CleverUniversity,” Int. J. Emerg. Trends Eng. Res., vol. 8, no. 5, pp. 1497-1502, 2020. https://doi.org/10.30534/ijeter/2020/04852020

[5] A. Bouchboua, R. Ouremchi, and M. Elghazi, "Educational Content Development Process in "CleverUniversity": Our Dynamic Adaptive Hypermedia Environment," Int. J. Emerg. Technol. Learn., vol. 11, no. 12, pp. 14-22, 2016. https://doi.org/10.3991/ijet.v11i12.5868

[6] A. Abdelkader, D. Souilem, and R. Braham, "E-Assessment System Based on IMS QTI for the Arabic Grammar,” Int. J. Adv. Comput. Sci. Appl., vol. 5, no. 10, pp. 92-100, 2014. https://doi.org/10.14569/ijacsa.2014.051013

[7] N. Hrich, M. Lazaar, and M. Khaldi, "Problematic of the assessment activity within adaptive E-learning systems," Int. J. Emerg. Technol. Learn., vol. 14, no. 17, pp. 133-142, 2019. https://doi.org/10.3991/ijet.v14i17.10675

[8] P. I. Wuisan and B. Wibawa, "Using electronic assessment system in higher education: Challenges and solutions for lectures, students and institutions," Int. J. Recent Technol. Eng., vol. 8, no. 2 Special Issue 9, pp. 673-678, 2019. https://doi.org/10.35940/ijrte.B11 41.0982S919

[9] Y. Lee, “Assessment Management System based on IMS QTI 2.1,” Int. J. Softw. Eng. Its Appl., vol. 8, no. 1, pp. 159-166, 2014.

[10] O. Sitthisak, L. Gilbert, M. Zalfan, and H. Davis, "Interactivity within ims learning design and question and test interoperability," in the 3rd International Conference on Web Information Systems and Technologies (WEBIST), 2007. https://doi.org/10.5220/00012693044 $\underline{00445}$

[11] G. Chemsi, M. Sadiq, M. Radid, and M. Talbi, "Formative E-assessment and behavioral commitment of students: Case of the Faculty of Science Ben M'sik," Int. J. Emerg. Technol. Learn., vol. 14, no. 12, pp. 4-14, 2019. https://doi.org/10.3991/ijet.v14i12.10389

[12] L. Cheniti-belcadhi, R. Braham, N. Henze, and W. Nejdl, "A generic framework for assessment in adaptive educational hypermedia," in Proceedings of IADIS International Conference, 2004, pp. 397-404. https://dblp.uni-trier.de/db/conf/iadis/icwi2004.html

[13] O. Auzende, H. Giroire, and F. Le Calvez, "A practical approach to using the IMS-QTI specification,” Int. J. Learn. Technol., vol. 7, no. 4, pp. 353-377, 2012. https://doi.org/10. 1504/IJLT.2012.052211

[14] S. B. Carol Fallon, "E-learning standards," Boca Raton, Fla.: St. Lucie Press, 2003, p. 25. https://doi.org/10.1201/9781420025330

[15] IMS GLS, "Igniting Digital Assessment Innovation." [Online]. Available: https://www.ims global.org/activity/qtiapip. [Accessed: 10-Apr-2020].

[16] B. Jérôme, T. Hoffmann, R. Howard, W. Kraan, M. McKell, and C. Smythe, "IMS Question and Test Interoperability (QTI): Overview Version 2.2," 2015. [Online]. Available: https://www.imsglobal.org/question/qtiv2p2/imsqti_v2p2_oview.html. [Accessed: 10-Apr2020].

[17] B. Jérôme, T. Hoffmann, R. Howard, W. Kraan, M. McKell, and C. Smythe, "IMS Question \& Test Interoperability: Implementation Guide Version 2.2," 2015. [Online]. Available: http://www.imsglobal.org/question/qtiv2p2/imsqti_v2p2_impl.html. [Accessed: 10Apr-2020].

[18] L. A. Alvarez-Gonzalez, A. Campos, M. E. De La Maza, and D. Ojeda, "Interactive assessment learning environment system under IMS-QTI specification," in 2014 International Conference on Education Technologies and Computers (ICETC), 2014, pp. 7-11. https ://doi.org/10.1109/ICETC.2014.6998894

[19] V. Gonzalez-Barbone and M. Llamas-Nistal, "EAssessment: Trends in content reuse and standardization," in 37th ASEE/IEEE Frontiers in Education Conference, 2007, pp. 11-16. https://doi.org/10.1109/FIE.2007.4417990

[20] F. Lazarinis, S. Green, and E. Pearson, "Measuring the conformance of hypermedia assessment tools to QTI," Int. J. Innov. Learn., vol. 6, no. 2, pp. 127-146, 2009. https://doi. org/10.1504/ijil.2009.022809 
[21] M. H. Zedan, A. Omran, and H. A. Hassan, "Test construction tool based on Interoperability standards," in 2014 International Conference on Engineering and Technology (ICET), 2014, pp. 1-7. https://doi.org/10.1109/ICEngTechnol.2014.7016815

[22] V. Tomberg and M. Laanpere, "Implementing Distributed Architecture of Online Assessment Tools Based on IMS QTI ver.2," in Handbook of Research on E-Learning Standards and Interoperability: Frameworks and Issues, 2011, pp. 41-58. https://doi.org/10.4018/9 78-1-61692-789-9

[23] P. Poulova, B. Klimova, and M. Krizek, "Selected e-learning systems and their comparison," in 2019 International Symposium on Educational Technology (ISET), 2019, pp. 297-300. https://doi.org/10.1109/ISET.2019.00070

[24] D. Vomvyras, A. Andreatos, and C. Douligeris, "Exam wizard: A novel e-assessment system," in 2019 4th South-East Europe Design Automation, Computer Engineering, Computer Networks and Social Media Conference (SEEDA-CECNSM), 2019, pp. 1-6. https:// doi.org/10.1109/SEEDA-CECNSM.2019.8908372

\section{Authors}

Mohammed Boussakuk is a PhD student at the Laboratory of Artificial Intelligence, Data Sciences and Emerging Systems in Sidi Mohamed Ben Abdellah University (USMBA), Fez, Morocco. His major research interests are adaptive and intelligent tutoring systems, computerized adaptive testing and e-learning.

Ahmed Bouchboua received his $\mathrm{PhD}$ in Computer Science in 2017 from Sidi Mohamed Ben Abdellah University Abdellah (USMBA) Fez, Morocco. His major research interests are adaptive and intelligent tutoring systems, computerized adaptive testing and e-learning. (ahmed.bouchboua@usmba.ac.ma)

Mohammed El ghazi is a professor in the Electrical Engineering Department of Superior School of Technology at the Sidi Mohamed Ben Abdellah University (USMBA), Fez, Morocco. He is a member of Laboratory of Artificial Intelligence, Data Sciences and Emerging Systems. (mohammed.elghazi@usmba.ac.ma)

Moulhime El bekkali is a professor in the National School of Applied Sciences at the Sidi Mohamed Ben Abdellah University (USMBA), Fez, Morocco. He is a member of Laboratory of Artificial Intelligence, Data Sciences and Emerging Systems. (moulhime.elbekkali @usmba.ac.ma)

Mohammed Fattah received his Ph.D. in Telecommunications and CEM at the University of Sidi Mohamed Ben Abdellah (USMBA) Fez, Morocco, 2011. He is a professor in the Electrical Engineering Department of the High school of technology at the Moulay Ismail University (UMI), Meknes, Morocco and he is a responsible of the research team 'Intelligent Systems, Networks and Telecommunications', IMAGE laboratory, UMI. (fattahm@gmail.com)

Article submitted 2020-06-16. Resubmitted 2020-08-19. Final acceptance 2020-08-20. Final version published as submitted by the authors. 